\title{
El uso del campus virtual para la tutorización y seguimiento del trabajo fin de grado: valoración del profesorado y propuesta de formación
}

\author{
Beatriz García-Antelo y Lorena Casal-Otero
}

\section{RESUMEN}

Este trabajo presenta un estudio de corte descriptivo en el que han participado 282 profesores/as de la Universidad de Santiago de Compostela (USC), España, y cuyo objetivo consistió en analizar el uso del campus virtual para realizar el seguimiento y tutorización de los Trabajos Fin de Grado (TFG). Para la recogida de información se hizo uso de un cuestionario elaborado ad hoc. Los resultados evidencian el bajo porcentaje de uso del campus virtual con este fin, argumentando la preferencia por la tutoría de carácter presencial y el desconocimiento de las posibilidades de esta herramienta. Consecuentemente, también se presenta una propuesta formativa dirigida a los/as docentes que deseen mejorar sus competencias en este campo.

Palabras clave: campus virtual, formación docente, tutores, trabajo académico, título de grado, España.

Beatriz García-Antelo de Pedagogía y Didáctica (Área MIDE) de la Facultad de Ciencias de la Educación, usc, España; directora del Centro de Tecnologías para el Aprendizaje y responsable del Programa de Formación e Innovación Docente de la usc. Temas de investigación: procesos de orientación y tutoría en la universidad, formación del profesorado, innovación en la educación superior.

\section{Lorena Casal-Otero}

Española. Doctora en Ciencias de la Educación, Universidad de Santiago de Compostela (USC), España. Profesora del Departamento de Pedagogía y Didáctica (Área Didáctica y Organización Escolar) de la Facultad de Ciencias de la Educación, usc, España. Temas de investigación: competencias para el uso de la tecnología, formación del profesorado para el uso de la tecnología, usos didácticos de las tecnologías, formación profesional para el empleo. 


\section{O uso do campus virtual para a orientação e acompanhamento do trabalho de fim de grau:} avaliação do professorado e proposta de formação

\section{RESUMO}

Este trabalho apresenta um estudo de corte descritivo no que participaram 282 professores/as da Universidade de Santiago de Compostela (USC), Espanha, e cujo objetivo consistiu em analisar o uso do campus virtual para realizar o acompanhamento e orientação dos Trabalhos de Fim de Grau (TFG). Para a recolhida de informação se utilizou um questionário elaborado ad hoc. Os resultados evidenciam a baixa porcentagem de uso do campus virtual com este fim, argumentando a preferência pela orientação de carácter presencial e o desconhecimento das possibilidades desta ferramenta. Consequentemente, também se apresenta uma proposta formativa dirigida aos/as docentes que desejam melhorar suas competências neste campo.

Palavras chave: campus virtual, formação docente, orientadores, trabalho acadêmico, diploma de grau, Espanha.

The use of the virtual campus for tutoring and monitoring the end-of-degree work: teachers' assessment and training proposal

\section{ABSTRACT}

This work presents a descriptive study in which 282 professors of the University of Santiago de Compostela (USC), Spain, have taken part. The objective of this study was to analyze the use of the virtual campus to monitor and supervise the end-of-degree projects (Trabajos de Fin de Grado). In order to gather the information, the authors made use of a questionnaire was used, specifically drawn up for this study. The results show a very low percentage for the use of the virtual campus for this purpose, evidencing the professors' preference for face-to-face tutoring and their ignorance of the possibilities provided by this tool. Consequently, the authors also present a training proposal for the teachers who want to improve their skills in this field.

Key words: virtual campus, teacher training, tutors, academic work, degree, Spain. 


\section{Introducción}

La configuración del Espacio Europeo de Educación Superior (EEES) ha traído consigo nuevos retos en las enseñanzas universitarias como son la introducción de metodologías de enseñanza-aprendizaje centradas en el estudiante o una nueva organización de los planes de estudio. Entre ellos también se encuentra el establecimiento del Trabajo Fin de Grado (TFG) como una materia de carácter obligatorio en el último curso de la titulación y que tiene por objeto la elaboración y defensa de un trabajo orientado a integrar las competencias adquiridas durante el grado. En España, el Real Decreto 1393/2007, que establece la ordenación de las enseñanzas universitarias oficiales (modificado parcialmente por el Real Decreto 861/2007), establece en su artículo 12 que las enseñanzas de grado concluirán con la elaboración y defensa de un TFG que debe estar orientado a la evaluación de competencias asociadas al título universitario y que tendrán un mínimo de seis créditos y un máximo del 12.5 por ciento del total de los créditos del mismo.

Esta amplia definición ha permitido a cada universidad delimitar de una forma más detallada las características de estos trabajos y su ajuste en los diferentes planes de estudio, dando lugar a normas específicas que regulan los procedimientos de desarrollo, presentación y evaluación de los mismos. Esta situación ha dado lugar a una gran heterogeneidad de enfoques en el número de créditos establecidos, el proceso de elección de tema, los criterios de evaluación, etcétera.

El carácter novedoso de un trabajo de estas características en la mayoría de titulaciones — dado que anteriormente a la creación del EEES no se realizaba - ha suscitado el acercamiento por parte de numerosos autores a algunos de los retos a los que se enfrentaban tanto las facultades como el alumnado y el profesorado: los mecanismos de gestión y coordinación (Civila, 2014; Elías, 2012; Martín et al., 2013), el proceso de tutorización (Derounian, 2011; Feather et al., 2014; Jamieson y Gray, 2006; Tood et al., 2006; Vera y Briones, 2015), las competencias adquiridas (Rekalde, 2011; Rullán et al., 2010; Zamora y Sánchez, 2014) o el procedimiento de evaluación (Mateo et al., 2012; Pascual y Pascual, 2012), entre otros.

Del mismo modo, en los últimos años un creciente número de investigaciones se han centrado en el uso del campus virtual (entendido como un entorno educativo que las universidades ponen a disposición del profesorado para dar soporte y apoyo a los procesos de enseñanza-aprendizaje) y las posibilidades de esta herramienta para gestionar, hacer seguimiento o tutorizar el TFG (Caro y Valverde, 2014; Sánchez et al., 2015; Esteban-Sánchez et al., 2016; Galiana et al., 2017; Serrano et al., 2017; Gómez et al., 2018). Estas investigaciones, realizadas en diferentes contextos, han puesto de manifiesto que las acciones de asesoramiento, seguimiento y acompañamiento que se realizan en el marco de la función tutorial se ven ampliadas y mejoran gracias al uso de la tecnología.

La asignatura TFG tiene rasgos especiales, tales como la ausencia de sesiones presenciales o programadas oficialmente (no hay clases magistrales al uso) y que cada alumno o alumna es guiado/a por un docente-tutor durante su realización (Esteban-Sánchez et al., 2016). Esta situación implica la necesidad de una metodología de trabajo diferente en la que el acompañamiento y la tutoría son elementos de gran relevancia en la materia, al considerarse un mecanismo principal en el desarrollo del proceso formativo.

En este sentido, una de las cuestiones que determina en gran medida el buen desarrollo del TFG reside en el proceso de tutorización, ya que aunque el alumnado es autónomo en su elaboración - realiza el trabajo partiendo de las competencias adquiridas a lo largo de la titulación y toma decisiones sobre este proceso-, es supervisado por un docente, que ejerce el rol de tutor/a, para que culmine con éxito su trabajo.

$\mathrm{El} / \mathrm{la}$ tutor/a ejerce dos funciones muy importantes: por un lado, asesora al alumnado de forma 
personalizada sobre su temática, por otro, aconseja sobre la organización y planificación del trabajo (Serrano et al., 2017). La tutoría se convierte así en un elemento de gran relevancia en la materia, al considerarse fundamental en el proceso formativo del alumnado, al mismo tiempo que un complemento elemental en la función docente del profesorado (Gómez et al., 2018).

Sin embargo, en la actualidad, e incluso con los requerimientos de asistencia al alumnado que el modelo de grados derivado del proceso de Bolonia propugna, es un hecho que el uso de la tutoría presencial en el despacho, e incluso virtual, a través de plataformas, es de escasa entidad y prácticamente inexistente (Tomeu-Hardasmal y Salguero-Hidalgo, 2018), lo que implica una dificultad en el seguimiento de la actividad de los estudiantes que repercute en el proceso de aprendizaje y en el proceso de construcción del TFG.

El uso de las tecnologías para hacer seguimiento del trabajo de los estudiantes y realizar tutorías se está consolidando en los últimos años. La posibilidad de generar escenarios en los que la ruptura espacio-temporal, la flexibilidad y la capacidad para comunicarse de forma ubicua, entre otros, facilitan los procesos de tutoría. En este sentido podemos afirmar que si en estos procesos de tutoría incluimos el uso de las Tecnologías de la Información y la Comunicación (TIC), hablaremos entonces de un modelo de acción tutorial más flexible y ajustado a las necesidades del alumnado (López y Prendes, 2017). En efecto, este tipo de tutoría no consiste simplemente en la utilización puntual y aislada de alguna herramienta concreta, sino que precisa del empleo de una variedad de recursos/entornos virtuales de comunicación a través de los cuales pueda enriquecerse el intercambio comunicativo y la propia función tutorial (Martínez et al., 2016).

En consecuencia, tenemos que poner de manifiesto que la función tutorial en entornos mediados por tecnología es compleja ya que requiere conocer las características de la tarea en sí, las acciones a realizar por el tutor y las herramientas necesarias, y es posible que no siempre los docentes poseen las actitudes para ello (Dellepiane, 2013). Por otro lado, la necesidad de competencias docentes vinculadas al uso de las tecnologías para la realización de seguimiento y tutoría no sólo exige manejar técnicamente un entorno virtual, sino también el uso didáctico de esas herramientas y ese tipo de competencias suelen ser limitadas (Casal, 2016). Es necesario, en este sentido, realizar procesos de adquisición de nuevos roles y nuevas competencias profesionales (Tejada y Pozos, 2018).

Además, cabe preguntarse si los docentes están suficientemente formados en habilidades comunicativas y de entrevista para dar respuesta a las diferentes necesidades y tipología de estudiantes en el marco de una tutoría integral (López y González, 2018).

\section{Método}

Los resultados que se muestran a continuación forman parte de una investigación más amplia de carácter descriptivo en la que se hizo uso de la técnica de encuesta y cuya finalidad se centra en conocer la percepción del profesorado de la Universidad de Santiago de Compostela (USC), España, sobre el desarrollo de la materia de Trabajo Fin de Grado. Para la recogida de información se elaboró un cuestionario ad hoc que engloba diferentes bloques de contenido: datos de identificación, relevancia y desarrollo del TFG, modalidades y formatos, competencias, evaluación, formación del profesorado y uso y potencialidades del campus virtual. Los resultados que se exponen en el presente artículo hacen referencia a este último bloque.

El cálculo de consistencia interna realizado a las escalas tipo Likert que componen el instrumento mediante el coeficiente alpha de Cronbach aportan un valor de 0.74 . El instrumento fue remitido online a los/as docentes de la USC. El análisis de los datos se realizó a través del paquete estadístico SPSS versión 20.0. 


\section{Objetivos}

- Conocer la percepción del profesorado de la Universidad de Santiago de Compostela sobre el uso del campus virtual (soportado en Moodle) para la tutorización y el seguimiento del TFG.

- Describir la propuesta formativa desarrollada desde el Programa de Formación e Innovación Docente de la USC respecto a las posibilidades que ofrece dicha herramienta para la gestión y tutorización de estos trabajos.

\section{Participantes}

Se remitió el instrumento en formato online al conjunto de la población, compuesta por el profesorado de la USC que en aquel momento era de 2053 . Tras un único envío se obtuvo la respuesta de 282 docentes procedentes de las diferentes áreas de conocimiento, con un mayor porcentaje de Ciencias Sociales y Jurídicas (31.9\%), seguido de Ciencias de la Salud (20.2\%), Ciencias Experimentales (19.5\%), Humanidades $(17.7 \%)$ y Enseñanzas Técnicas $(10.6 \%)$. El porcentaje de hombres $(53.9 \%)$ que han respondido al cuestionario es ligeramente superior que el de mujeres (46.1\%). En cuanto a la representación por campus, proceden mayoritariamente del de Santiago (79.8\%) frente al de Lugo (20.2\%).

En cuanto a los centros en que desarrollan su labor docente, cabe señalar que han participado en el estudio profesores/as de todos los centros de la USC, con un mayor porcentaje de los siguientes: Facultad de Filología (9.9\%), Facultad de Ciencias de la Educación $(9.2 \%)$, Escuela Politécnica Superior (6.7\%), Facultad de Psicología (6.0\%), Escuela Politécnica Superior de Ingeniería (5.3\%), Facultad de Ciencias Económicas y Empresariales (5.3\%) y Facultad de Derecho (5.3\%).

\section{Resultados}

\section{Percepción del profesorado de la} Universidad de Santiago de Compostela sobre el uso del campus virtual para la coordinación y el seguimiento del TFG

Los resultados obtenidos ponen de manifiesto que un bajo porcentaje de encuestados/as (16.0\%) hacen uso del campus virtual en el proceso de coordinación y seguimiento del TFG (figura 1).

\section{Figura 1. Uso del campus virtual para el desarrollo del TFG}

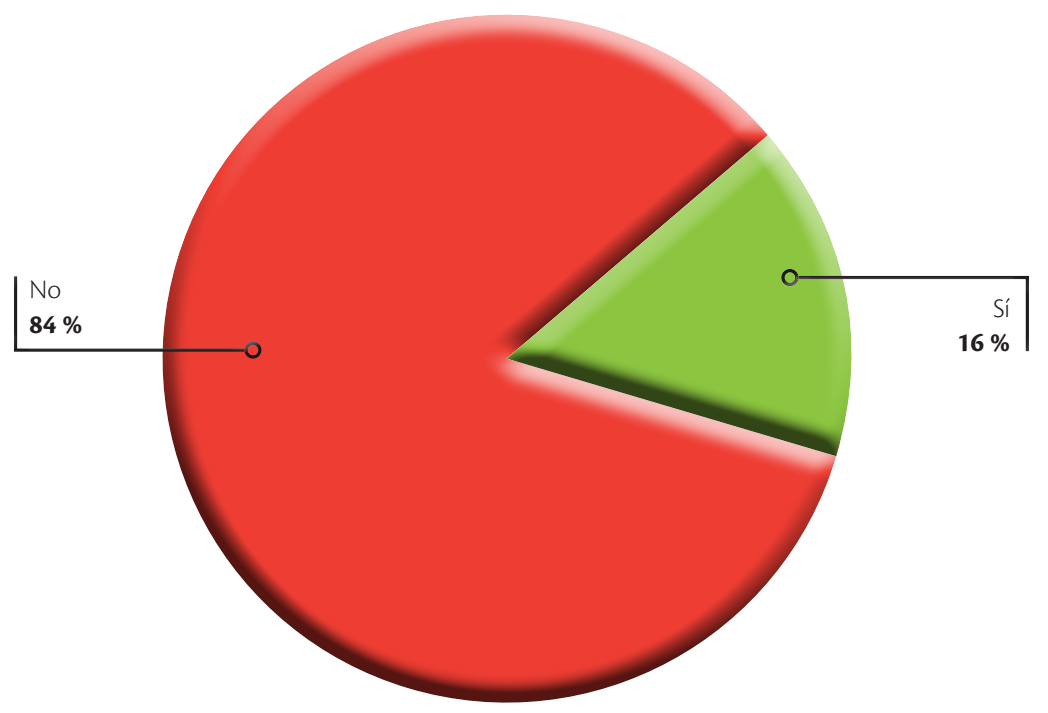


De ese porcentaje que sí indica haber hecho uso del campus virtual, lo hacen mayoritariamente para acercar documentación necesaria en el desarrollo del TFG (normativa, calendario, listado de tutores/as, etcétera) y para el envío de avisos generales y anuncios al alumnado a través del foro. A continuación, casi la mitad de encuestados/as también afirman dar bastante o mucho uso a esta herramienta para responder dudas y cuestiones de carácter general sobre aspectos organizativos del TFG.
Por el contrario, son menos los/as docentes que acuden al campus virtual para la comunicación privada a través del foro con los/as estudiantes que tutorizan o para la coordinación interna entre los tutores/as.

En último lugar cabe señalar que mientras casi la mitad de profesorado $(42.2 \%)$ señala que usa bastante o mucho el campus para comunicarse con el alumnado a través de la mensajería interna y para intercambiar ficheros de forma privada con él, un porcentaje similar $(46.7 \%)$ indica justo lo contrario, que hace ningún o poco uso de esta herramienta.

Tabla 1. Frecuencia de uso del campus virtual

\begin{tabular}{|c|c|c|c|c|c|c|c|}
\hline \multirow{2}{*}{ Ítems } & \multicolumn{2}{|c|}{$\begin{array}{l}\text { Estadísiticos } \\
\text { descriptivos }\end{array}$} & \multicolumn{5}{|c|}{ Porcentajes } \\
\hline & Media & $\begin{array}{l}\text { Desv. } \\
\text { típica }\end{array}$ & Nada & Poco & Regular & Bastante & Mucho \\
\hline $\begin{array}{l}\text { Acercar documentación (normativa, calendario, listado de } \\
\text { tutores/as, etcétera) }\end{array}$ & 3.89 & 1.133 & 4.4 & 8.9 & 15.6 & 35.6 & 35.6 \\
\hline $\begin{array}{l}\text { Responder dudas y preguntas generales sobre aspectos } \\
\text { organizativos del TFG. }\end{array}$ & 3.27 & 1.286 & 13.3 & 11.1 & 31.1 & 24.4 & 20.0 \\
\hline $\begin{array}{l}\text { Coordinación interna de los/as tutores/as (utilizando } \\
\text { herramientas ocultas para los/as estudiantes). }\end{array}$ & 2.31 & 1.311 & 35.6 & 26.7 & 17.8 & 11.1 & 8.9 \\
\hline $\begin{array}{l}\text { Enviar avisos generales y anuncios al alumnado a través } \\
\text { del foro. }\end{array}$ & 3.67 & 1.331 & 13.3 & 4.4 & 15.6 & 35.6 & 31.1 \\
\hline $\begin{array}{l}\text { Comunicarme, de forma privada, con el alumnado que } \\
\text { tutorizo a través de la mensajería interna. }\end{array}$ & 2.93 & 1.572 & 26.7 & 20.0 & 11.1 & 17.8 & 24.4 \\
\hline $\begin{array}{l}\text { Comunicarme, de forma privada, con el alumnado que } \\
\text { tutorizo a través del foro. }\end{array}$ & 2.33 & 1.430 & 37.8 & 28.9 & 8.9 & 11.1 & 13.3 \\
\hline $\begin{array}{l}\text { Intercambiar ficheros, de forma privada, con el/la } \\
\text { estudiante. }\end{array}$ & 2.93 & 1.543 & 26.7 & 17.8 & 13.3 & 20.0 & 22.2 \\
\hline
\end{tabular}

Fuente: elaboración propia. 
A través de una pregunta abierta se consultó al profesorado que señalaba no hacer uso del campus virtual en el desarrollo del TFG cuáles eran los motivos por los que no lo hacía. Tras el análisis de contenido de las respuestas podemos indicar que de éstas la razón principal es el uso prioritario de la tutoría presencial, entendiéndola como suficiente en el proceso de tutorización de este tipo de trabajos. Algunos de los argumentos aportados en esta línea son los siguientes:

Porque la tutorización tiene que ser presencial y corrigiendo una y otra vez los sucesivos borradores que nos entregan.

Porque normalmente la tutorización del TFG abarca sólo uno o dos alumnos al año. Por lo tanto es suficiente la tutorización presencial.

La comunicación virtual pervierte el mensaje y la transmisión. La presencialidad y el contacto personal y directo con el tutor es imprescindible.

Uso el campus virtual para las otras materias, pero en el TFG prefiero un contacto más directo y presencial.

No hago uso del campus virtual, porque considero que la tutorización tiene que ser presencial.

Son TFG que requieren de una tutorización personal donde el alumno explique las dificultades y problemas de un modo que le campus virtual no permite.

Porque es más cómodo y funcional tener un contacto directo con el alumno/a.

La corrección es más fácil si se hace de modo presencial en un trabajo de este tipo, que requiere mayor atención y exhaustividad.

Considero mucho más efectiva y eficiente la tutorización presencial en el caso de los TFG.

Otro grupo de docentes $(18.1 \%$ del total) señala que no considera necesario el uso del campus virtual dado que realiza procesos de comunicación asíncrona con el alumnado a través del correo electrónico.

Atiendo uno o dos tfg, de contenidos distintos, así que me resulta mucho más rápido y operativo usar otros medios, como el e-mail o los encuentros en tutoría presencial"

En la práctica los alumnos concretan a través de e-mail todos los asuntos: citas con el tutor, dudas, envío de borradores, etcétera.

Me resulta más cómodo contactar con el alumnado por medio del correo electrónico.

No me parece necesaria la comunicación, con el alumnado es personal o mediante correo electrónico.

No lo considero necesario [...] En caso en que no exista (períodos vacacionales...) ese contacto lo suplimos con el correo electrónico y herramientas en la nube.

El correo electrónico y las tutorías presenciales fueron, hasta ahora, suficientes.

La comunicación entre los alumnos tutorizados y yo se realiza de forma fluida y rápida por correo electrónico institucional.

Finalmente, otro de los argumentos del profesorado hace referencia a su desconocimiento de las posibilidades que ofrece esta herramienta, aludiendo a su falta de formación al respecto. En este sentido se pronuncian algunos de ellos:

Lo uso habitualmente para las materias de grado y máster, pero carezco de información y formación para saber cómo usar eficazmente el campus virtual en el caso de los TFG.

El uso que se le daría es semejante al de grupo de correo electrónico (mensajes), por lo que la incertidumbre y desconocimiento de otras herramientas del campus virtual hace que se desperdicie esta oportunidad. 


\section{Experiencia formativa desarrollada desde el Programa de Formación e Innovación Docente (PFID) de la usc respecto a las posibilidades que ofrece el campus virtual para la gestión y tutorización de estos trabajos}

En el curso 2013-2014 se empieza a utilizar la plataforma Moodle como soporte al campus virtual de la Universidad de Santiago de Compostela (USC). A partir de ese momento se ha ido diversificado su funcionalidad, ampliando el uso de las aulas virtuales como espacios en los que exclusivamente se alojan materiales y avanzando hacia usos que ayudan a completar procesos formativos.

A ese avance ha contribuido el impulso realizado desde el Programa de Formación e Innovación Docente de la USG y, en efecto, desde el curso 20132014 se apostó por un programa formativo dirigido al profesorado universitario que estuviera centrado en la adquisición de competencias para el uso diversificado de las herramientas del campus virtual, centrándose en el componente didáctico y partiendo de las necesidades del profesorado como eje sobre el que girara cualquier acción de formación.

Partiendo de estas premisas, en el curso 20142015 se inició una propuesta formativa de carácter semipresencial dirigida a la gestión y uso didáctico del aula virtual en esta asignatura cuya denominación es "Trabajos Fin de Grado: la gestión eficiente del aula virtual", con una duración de cuatro horas (tres presenciales y una online). Su finalidad radica en potenciar que en las aulas virtuales de la asignatura de TFG se realicen procesos encaminados a favorecer intercambios comunicativos, de seguimiento y tutorización que prioricen la atención personalizada a los estudiantes.

Más concretamente, los objetivos de esta acción formativa son los siguientes:

- Identificar herramientas y posibilidades de gestión de los estudiantes en el aula virtual.
- Conocer las posibilidades del trabajo con grupos y agrupamientos.

- Aprender a crear grupos y agrupamientos (diferentes modalidades de trabajo con grupos en la plataforma Moodle).

- Conocer las diferentes formas de asignar estudiantes a los grupos.

- Asignar recursos y actividades a grupos y agrupamientos.

- Configurar la actividad "Tarea": uso eficiente como herramienta para la gestión de trabajos.

Para el logro de dichos objetivos se propone el trabajo en torno a los siguientes contenidos:

- Introducción: posibilidades de uso de las aulas virtuales de TFG.

- La gestión de los usuarios en el aula virtual: procesos y posibilidades.

- Posibilidades y uso de la herramienta "grupos".

- Posibilidades y uso de la herramienta "agrupamientos".

- Las posibilidades de uso de los grupos en diferentes actividades: "Foro" y "Tarea".

- Actividad "Foro" y actividad "Tarea": posibilidades de usos, configuración y gestión.

En la sesión presencial se realizan diferentes actividades dirigidas a conocer las posibilidades de uso de las aulas virtuales de la asignatura y a implementar las opciones y herramientas presentadas. Tras esta sesión y de forma individual, cada docente realiza una tarea en su aula virtual de pruebas centrada en la implementación de las opciones trabajadas.

Hasta el momento actual se han impartido nueve acciones formativas, cinco de ellas en el campus de Santiago y cuatro en el campus de Lugo. La demanda por parte del profesorado fue elevada, superando en casi todas las ediciones ofertadas el número de inscritos/as las plazas disponibles. En total asistieron 
112 docentes procedentes de diferentes áreas de conocimiento.

\section{Discusión y conclusiones}

El rol de tutorización que desempeña el docente exige, entre otros, el desarrollo de estrategias de comunicación con el alumno/a durante todo el proceso. El campus virtual, cuyo uso está ampliamente extendido entre el profesorado, supone una herramienta de utilidad para el seguimiento y tutorización del TFG en tanto que facilita la comunicación síncrona y asíncrona entre el profesorado y el alumnado a través del correo electrónico, el foro, etcétera. Actualmente en la USC se crean automáticamente en el campus virtual aulas por cada una de las materias de TFG de las diferentes titulaciones, hecho que a priori debería favorecer su uso por parte del profesorado y el alumnado.

No obstante, respecto a los datos analizados en nuestra investigación, cabe decir que el profesorado no hace uso de las potencialidades del campus virtual para realizar actividades de seguimiento y tutorización del TFG de los estudiantes. Las razones aportadas por los docentes para no usar el campus virtual aluden a cuestiones como la preferencia de la tutoría de carácter presencial, entendiéndola como fundamental en el proceso de asesoramiento de un trabajo de estas características así como al desconocimiento de lo que puede aportar esta herramienta al proceso de coordinación y tutorización.

Lo dicho hasta ahora corrobora los resultados obtenidos en otras investigaciones que previamente habían puesto de manifiesto el bajo porcentaje de uso del campus virtual para realizar actividades de seguimiento y tutoría (Tomeu-Hardasmal y Salguero-Hidalgo, 2018). Otros autores (Muñoz-Gea et al., 2016) informan que el desconocimiento de las posibilidades de la plataforma para dar soporte a procesos de acompañamiento y tutoría del TFG es una cuestión fundamental y, además, la enorme cantidad de datos en la plataforma está claramente infrautilizada, entre otros aspectos, debido a la falta de formación específica del profesorado.

En este último punto, se ha considerado necesaria la intervención desde el Programa de Formación e Innovación Docente de la USC, desarrollando una acción formativa destinada a aquellos/as profesores/as que muestren interés por ampliar sus competencias al respecto. No podemos olvidar que el carácter diferenciador y novedoso del Trabajo Fin de Grado respecto del de otras materias hace necesario el uso adaptado de las herramientas del campus virtual a sus características, lo que requiere de una formación específica sobre cuestiones como la gestión de usuarios, el uso de la herramienta grupos y agrupamientos, el foro, la actividad "tarea", etcétera.

La elevada participación del profesorado en las nueves ediciones de esta acción formativa pone de manifiesto su interés por ampliar y mejorar su formación respecto a esta temática y se vincula a uno de los motivos que argumenta el profesorado encuestado para no realizar un mayor aprovechamiento del campus virtual: el desconocimiento de las posibilidades que éste puede ofrecer para la tutorización de los trabajos fin de grado.

De acuerdo con esto y en línea con otros autores (Dellepiane, 2013), consideramos que una mayor formación en competencias para el uso didáctico del campus virtual puede beneficiar el aprovechamiento que el profesorado realiza del mismo para dar soporte a la asignatura TFG, ya que Moodle dispone de herramientas que nos permiten realizar seguimiento sistemático de la actividad del estudiante, ofrecer retroalimentación e implementar procesos de tutoría electrónica en el aula virtual de la asignatura. No debemos olvidar tampoco la importancia del diseño de esta formación, que debe ir más allá de la formación instrumental y centrarse en las posibilidades que ofrece la dialéctica entre tecnología, pedagogía y disciplina (Cejas-León y Navío Gámez, 2018), que es considerado un aspecto fundamental para facilitar la transferencia y transformar prácticas docentes. 


\section{Referencias}

Caro, M.T. y M. T. Valverde (2014), "Aprendizaje bimodal de las competencias comunicativa y digital en contextos formales de Educación superior: la realización procesual de trabajos Fin de Grado en Moodle", Revista Internacional de Aprendizaje y Cibersociedad, vol. 18, núm. 1, pp. 43-53, <https://journals.epistemopolis. org/index.php/textos/article/view/1142/700>

Casal, L. (2016), "Competencias TIC del profesorado participante en los planes de formación gestionados por los agentes sociales: el Sindicato FETE-UGT", Tesis doctoral, Santiago de Compostela, Universidad de Santiago de Compostela.

Cejas-León, R. y A. Navío Gámez (2018), "Formación en TIC del profesorado universitario. Factores que influyen en la transferencia a la función docente", Profesorado. Revista de Currículum y Formación de Profesorado, vol. 22, núm. 3, pp. 271-293, DOI: 10.30827/profesorado. v22i3.8002.

Civila, A. (2014), "Gestión de unos 800 trabajos fin de grado en una facultad de Ciencias de la Educación", en K. Pérez, B. Bilbao, E. Fernández de Larrea, B. Otero y P. Ruiz de Gauna (coords.), Retos y oportunidades del TFG para la sociedad del conocimiento (Libro de Actas del I Congreso Interuniversitario del Trabajo Fin de Grado), pp. 356-365, Bilbao, Universidad del País Vasco.

Dellepiane, P. (2013), "Las tutorías en la universidad: propuesta de formación en entornos virtuales de aprendizaje", Campus Virtuales, vol. 2, núm. 2, pp. 66-74, <http://uajournals.com/ojs/index.php/ campusvirtuales/article/view/39/38>.

Derounian, J. (2011), "Shall we dance? The importance of staff-student relationships to undergraduate dissertation preparation", Active Learning in Higher Education, vol. 12, núm. 2, pp. 91-100, DOI: https:// doi.org/10.1177/1469787411402437.

Elías, A. (2012), "Algunas reflexiones en clave de gestión tras las primeras defensas de los Trabajos Fin de Grado", Revista Andaluza de Relaciones Laborales, vol. 25-26, pp. 113-134.
Esteban-Sánchez, A.L., J. Esteban-Escaño y M. L. SeinEchaluce (2016), "Seguimiento adaptativo del Trabajo de Fin de Grado en ingeniería con apoyo de Moodle", VAEP-RITA, vol. 4, núm. 2, pp. 65-72, <https:// repositorio.grial.eu/ bitstream/ grial/ 675/ 1 /201606-uploads-VAEP-RITA.2016.V4.N2.A4.pdf>.

Feather, D., J. R. Anchor y C. J. Cowton (2014), "Supervisors' perceptions of the value of the undergraduate dissertation", The International Fournal of Management Education, vol. 12, núm. 1, pp. 14-21, DOI: https://doi.org/10.1016/j.ijme.2013.06.002.

Galiana, A., N. Torres, C. Jordi, M. T. Franquet, R. Torrens y A. Pigrau (2017), "La gestión y evaluación del trabajo de fin de grado a través de Moodle 3.0: una experiencia en la Facultad de Ciencias Jurídicas URV”, en A. M. Delgado e I. Beltrán (coords.), Las TIC y las buenas prácticas en la docencia del derecho, Barcelona, Huygens, pp. 211-223.

Gómez, M. E., R. Serrano, M. I. Amory C. Huertas(2018), "Los trabajos de fin de grado (TFG) como innovación en el EEES. Una propuesta de tarea colaborativa basada en la tutoría piramidal", Educar, vol. 54, núm. 2, pp. 369-389, <https://www.raco.cat/index.php/ Educar/article/view/338177/429046>.

Gobierno de España (2007), Real Decreto 1393/2007, de 29 de octubre, por el que se establece la ordenación de las enseñanzas universitarias oficiales, Ministerio de Educación y Ciencia, Boletín Oficial del Estado, $n^{\circ}$ 260, 30 de octubre de 2007, disponible en: <https:// www.boe.es/buscar/pdf/2007/BOE-A-2007-18770consolidado.pdf $>$.

Jamieson, S. y C. Gray (2006), "The supervision of undergraduate research students: expectations of student and supervisor", Practice and Evidence of Scholarship of Teaching and Learning in Higher Education, vol. 1, núm. 1, pp. 37-59, <http://community.dur. ac.uk/pestlhe.learning/index.php/pestlhe/article/ view/125/141>.

López,I.yP.González(2018), "Latutoríauniversitariacomo 
espacio de relación personal. Un estudio de caso múltiple", Revista de Investigación Educativa, vol. 36, núm. 2, pp. 381-399, DOI: http://dx.doi.org/10.6018/ rie.36.2.291161.

López, P. y M. P. Prendes (2017), "Estudio longitudinal sobre tutoría académica flexible en la universidad", Profesorado. Revista de Currículumy Formación del Profesorado, vol. 21, núm. 4, pp. 259-258, <https://recyt.fecyt.es/ index.php/profesorado/article/view/62504>.

Martín, P.J., M. A. Renarty M. G. Puigcerver(2013), “Los beneficios de la coordinación horizontal en la realización de los TFG para los alumnos del grado en Administración y Dirección de Empresas", Revista de Investigación en Educación, vol. 1, núm. 11, pp. 97-117, $<$ http://reined.webs.uvigo.es/index.php/reined/ article/view/164>.

Martínez, P., J. Pérez y M. Martínez (2016), "Las TIC y el entorno virtual para la tutoría universitaria", Educación XXI, vol. 19, núm. 1, pp. 287-310, DOI: 10.5944/educXX1.13942.

Mateo, J., A. Escofet, F. Martínez, J. Ventura y D. Vlachopoulos (2012), "The final year project (FYP) in social sciencies: establishment of its associated competences and evaluation standards", Studies in Educational Evaluation, núm. 38, pp. 28-34, DOI: 10.1016/j.stueduc.2011.12.002.

Muñoz-Gea, J. P., F. J. Pérez, S. Busquier, M. M. Silva y C. Angosto (2016), "Interacción de los estudiantes con las actividades de Moodle: un estudio basado en web mining", Revista Internacional de Ciencia Tecnología y Sociedad, vol. 5, núm. 1, pp. 59-68, DOI: https://doi. org/10.18848/2474-588X/CGP/v05i01/59-68.

Pascual, M. y M. M. Pascual (2012), "Propuesta de evaluación del Trabajo Fin de Grado en Derecho", Aula Abierta, vol. 40, núm. 1, pp. 85-102.

Rekalde, I. (2011), “CCómo afrontar el Trabajo Fin de Grado? Un problema o una oportunidad para culminar con el desarrollo de las competencias", Revista Complutense de Educación, vol. 22, núm. 2, pp 179-193, <http://revistas.ucm.es/index.php/RGED/ article/view/38488/37226>.
Rullán, M., M. Fernández, G. Estapè y M. D. Márquez (2010), "La evaluación de competencias transversales en la materia trabajos fin de grado. Un estudio preliminar sobre la necesidad y oportunidad de establecer medios e instrumentos por ramas de conocimiento", REDU, Revista de Docencia Universitaria, vol. 8, núm. 1, pp. 74100, DOI: https://doi.org/10.4995/redu.2010.6218.

Sánchez, F., J. García, D. López, M. Alier, J. Cabré, H. García y E. Vidal (2015), "El método socrático como guía del trabajo de fin de grado", ReVisión, vol. 8, núm. 1, pp. 53-62, <http://www.aenui.net/ojs/index. php? journal $=$ revision \&page $=$ article $\&$ op $=$ viewArtic le\&path $\% 5 \mathrm{~B} \% 5 \mathrm{D}=142>$.

Serrano, R., C. Huertas, M. Osuna, M. Rosas, A. Sánchez, M. Sánchez, I. Pérez, P. Torres, D. F. Palacios y D. Murrillo (2017), "La tutoría piramidal como estrategia de aprendizaje para el diseño y desarrollo del trabajo final de grado en educación", Revista de Innovación y Buenas Prácticas Docentes, núm. 3, DOI: https://doi.org/10.21071/ripadoc.v3i0.9966.

Tejada, J. y K. Pozos (2018), "Nuevos escenarios y competencias digitales docentes: hacia la profesionalización docente con TIC", Profesorado. Revista de Currículum y Formación del Profesorado, vol. 22, núm. 1, pp. 25-51, <https://recyt.fecyt.es/index.php/ profesorado/article/view/63620/pdf_60>.

Tomeu-Hardasmal, A.J., y A. G. Salguero-Hidalgo (2018), "Un modelo innovador de tutoría, adaptado a las necesidades individuales del estudiante", Revista Iberoamericana de Informática Educativa, núm. 27, pp. 1-12, <http://161.67.140.29/iecom/index.php/IECom/ article/view/303/295>.

Tood, M., K. Smith y P. Bannister (2006), "Supervising a social science undergraduate dissertation: staff experiences and perceptions", Teaching in Higher Education, vol. 11, núm. 2, pp. 161-173, DOI: https:// doi.org/10.1080/13562510500527693.

Vera, J. y E. Briones (2015), "Perspectiva del alumnado de los procesos de tutorización y evaluación de los trabajos de fin de grado", Cultura y Educación, vol. 27, núm. 4, pp. 726-765, DOI: 10.1080/11356405.20 15.1089391. 
Zamora, F. y J. Sánchez (2014), "Los Trabajos Fin de Grado: una herramienta de desarrollo de competencias transversales en la Educación Superior", REDU, Revista de Docencia Universitaria, vol. 13, núm. 3, pp. 197-211, DOI: https://doi.org/10.4995/redu.2015.5426.

\section{Cómo citar este artículo:}

García-Antelo Beatriz, y Lorena Casal-Otero (2020), "El uso del campus virtual para la tutorización y seguimiento del trabajo fin de grado: valoración del profesorado y propuesta de formación", Revista Iberoamericana de Educación Superior (RIES), vol. XI, núm. 32, pp. 216-227, DOI: https://doi.org/10.22201/iisue.20072872e.2020.32.823 [Consulta: fecha de última consulta]. 